\title{
Odd-even effect dependence on the excitation energy in low energy fission
}

\author{
M. Mirea ${ }^{\mathrm{a}}$ \\ Horia Hulubei National Institute for Physics and Nuclear Engineering, PO Box MG-G, Bucharest, Romania
}

\begin{abstract}
An inversion of the odd-even effect was observed experimentally in cold fission: the odd-odd fragmentation yields are favored over the even-even ones for excitations energies of the fragments smaller than $4 \mathrm{MeV}$. This effect is linked to the important problem of quasiparticle excitations during the dynamical evolution of the nuclear system from its ground-state configuration up to scission. An explanation based on the Landau-Zener promotion mechanism generalized for superfluid systems is offered for the inversion of the odd-even effect. In principle, the even-even fission products cannot be produced at very low excitation energies due dynamical quasiparticle excitations produced in the avoided- level-crossing regions. These excitations are produced with a large probability when the nuclear system deforms slowly.
\end{abstract}

\section{Introduction}

In the low energy fission, the mass and charge distributions of fragments show a preference for even numbers of neutrons and protons [1-5]. When the excitation energy increases, the magnitude of this odd-even effect is lowered [6]. Surprisingly, in the case of cold fission, where the excitation energy of the fragments is so small that no neutrons can be emitted, an inversion of the oddeven effect is produced. As evidenced experimentally $[7,8]$ for $\mathrm{U}$ isotopes, in the cold region regime the oddodd fragmentations dominate at excitations energies lower than $4-6 \mathrm{MeV}$. This behavior is typical, being observed also for the fission of $\mathrm{Cf}[9,10]$. This inversion can be explained statistically by involving the level densities of odd and even nuclei $[11,12]$. This effect should be linked to the important problem of single particle excitations along the dynamical evolution of the nuclear system in a large scale collective amplitude motion. In principle, such excitations can be produced by the Landau-Zener promotion mechanism $[13,14]$.

\section{The landau-zener effect and the seniority mixing}

Two single particle levels having the same good quantum numbers associated to some symmetries of the system cannot in general intersect and exhibit avoided level crossing regions. An interaction $h_{i j}$ is always present in an avoided crossing region between the two single particle states of energies $\epsilon_{i}$ and $\epsilon_{j}$. For a slowly deforming nuclear system, a single particle will remain on the same level after the passage of an avoided crossing region. For a rapid variation of the deformation parameter, the nucleon will skip with a large probability from one single particle level to the other one after the passage of such a pseudocrossing. We say that the motion is diabatic. It

a e-mail: mirea@ifin.nipne.ro is possible to know the final probabilities of occupation of a single particle level after the passage through an avoided crossing level region by solving a set of time dependent coupled channel equations. These equations are derived from the time dependent Schrödinger equation. The promotion mechanism of a nucleon from one level to another is known as the Landau-Zener effect. It describes the non adiabatic transitions in avoided level crossing regions between potential curves or energy levels.

The behavior of the Landau-Zener effect in the case of superfluid systems will be derived. It is possible that only one nucleon of a pair skips to the another level producing a dynamical quasiparticle excitation in an avoided crossing region. Three situations depicted in Fig. 1 are possible. In the first case, the pairs remain on the same single particle level after the passage through the avoided level crossing region. In the second case, a pair destruction is illustrated. In the last case, two fermions generates a pair. By using quasiparticle creation $\alpha_{i}^{+}$and annihilation operators $\alpha_{i}$ that act on a superposition of seniority zero and seniority two wave functions, we construct some interactions able to break or to create a pair when the system traverses an avoided level crossing region. This kind of perturbations were postulated in a previous work where the Landau-Zener effect was generalized in the superfluid seniority one systems [15]. The equations that governs the pair breaking mechanism are directed by the variational principle $[16,17]$. The residual perturbation in the avoided level crossing region is:

$$
\begin{aligned}
H^{\prime}(t)=\sum_{i, j \neq i}^{n} h_{i j}(t) & {\left[\alpha_{i(0)} \alpha_{\bar{j}(0)} \prod_{k \neq i, j} \alpha_{k(0)} \alpha_{k(i j)}^{+}\right.} \\
& \left.+\alpha_{i(0)}^{+} \alpha_{\bar{j}(0)}^{+} \prod_{k \neq i, j} \alpha_{k(i j)} \alpha_{k(0)}^{+}\right],
\end{aligned}
$$

where $h_{i j}$ is the interaction energy in the avoided crossing regions.

The Hamitonian with paring cannot give integers numbers of neutrons and protons of the fragments at 


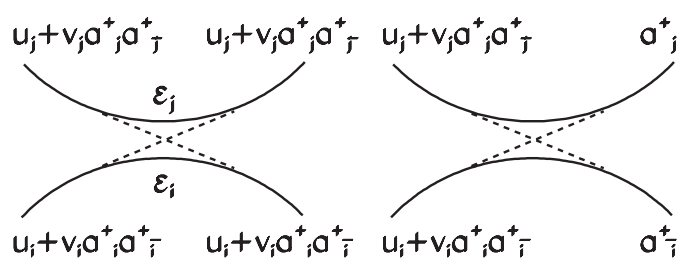

(a) (b)

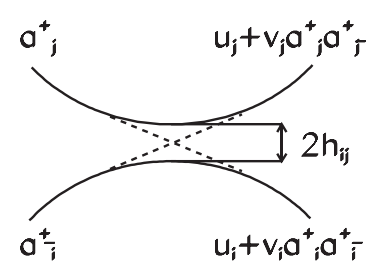

(c)

Figure 1. (a) No change of configuration after the passage through the avoided crossing region; (b) a pair is destroyed; (c) a pair is formed [16].

scission. Therefore we also implemented an recipe for a dynamical projection of the particle numbers of the two fragments. At scission, the two fission fragments must be characterized by a supplementary condition

$$
\left|N_{2} \hat{N}_{1}-N_{1} \hat{N}_{2}\right|=0
$$

where $N_{1}$ and $N_{2}$ are the number of particles in the final fragments labeled 1 and 2, respectively, and

$$
\hat{N}_{1}=\sum_{k 1}\left(a_{k_{1}}^{+} a_{k_{1}}+a_{\bar{k}_{1}}^{+} a_{\bar{k}_{1}}\right) ; \hat{N}_{2}=\sum_{k 2}\left(a_{k_{2}}^{+} a_{k_{2}}+a_{\bar{k}_{2}}^{+} a_{\bar{k}_{2}}\right)
$$

are the corresponding operators. Here, $k_{1}$ and $k_{2}$ run over the pairing active level states that are located in the potential wells of the final fragment 1 and of the final fragment 2, respectively. The condition (1) can be introduced in the equations of motion by means of the Lagrange multipliers $[18,19]$.

As mentioned, the microscopic equations of motion emerge from the variational principle with the energy functional

$$
\delta \mathcal{L}=\delta\left\langle\varphi\left|H+H^{\prime}-\lambda\right| N_{2} \hat{N}_{1}-N_{1} \hat{N}_{2}\left|-i \hbar \frac{\partial}{\partial t}\right| \varphi\right\rangle .
$$

The trial many-body function $\varphi$ is considered as a superposition of Bogoliubov seniority zero and seniority two wave functions

$$
\begin{aligned}
|\varphi(t)\rangle= & {\left[c_{0} \prod_{k}\left(u_{k(0)}(t)+v_{k(0)}(t) a_{k}^{+} a_{\bar{k}}^{+}\right)\right.} \\
& +\sum_{j, l \neq j} c_{j l}(t) a_{j}^{+} a_{\bar{l}}^{+} \\
& \left.\times \prod_{k \neq j, l}\left(u_{k(j l)}(t)+v_{k(j l)}(t) a_{k}^{+} a_{\bar{k}}^{+}\right)\right]|0\rangle .
\end{aligned}
$$

$c_{0}$ is the amplitude of the seniority zero wave function while $c_{j l}$ are amplitudes for the seniority two wave functions for configurations in which the single particle orbitals $j$ and $l$ in the active pairing space are each blocked by only one unpaired nucleon. Here, the vacancy $u_{k}$ and occupation $v_{k}$ amplitudes are not the adiabatic solutions of the BCS equations and depend on the variation in time of the generalized parameters and the history of the nuclear system. $\lambda$ is a Lagrange multiplier. The next coupled channel equations are obtained, eventually:

$$
\begin{gathered}
i \hbar \dot{\rho}_{k(0)}=\kappa_{k(0)} \Delta_{k(0)}^{*}-\kappa_{k(0)}^{*} \Delta_{k(0)}, \\
i \hbar \dot{\rho}_{k(j l)}=\kappa_{k(j l)} \Delta_{k(j l)}^{*}-\kappa_{k(j l)}^{*} \Delta_{k(j l)}, \\
i \hbar \dot{\kappa}_{k(0)}=\left(2 \rho_{k(0)}-1\right) \Delta_{k(0)}+2 \kappa_{k(0)}\left(\epsilon_{k}-s N_{i_{k}} \lambda\right) \\
-2 G_{k k} \rho_{k(0)} \kappa_{k(0)},
\end{gathered}
$$

$$
\begin{aligned}
& i \hbar \dot{\kappa}_{k(j l)}=\left(2 \rho_{k(j l)}-1\right) \Delta_{k(j l)}+2 \kappa_{k(j l)}\left(\epsilon_{k}-s N_{i_{k}} \lambda\right) \\
&\left.-2 G_{k k} \rho_{k(j l)} \kappa_{k(j l)}\right) \\
& i \hbar \dot{P}_{0}=\sum_{l, j \neq l} h_{l j}\left(S_{0 j l}^{*}-S_{0 j l}\right) \\
& i \hbar \dot{P}_{j l}=h_{l j}\left(S_{0 j l}-S_{0 j l}^{*}\right) \\
& i \hbar \dot{S}_{0 j l}=S_{0 j l}\left(\bar{E}_{0}-\bar{E}_{j l}\right)+S_{0 j l}\left(\Sigma_{k \neq j, l} T_{k(j l)}-\Sigma_{k} T_{k(0)}\right) \\
&+\Sigma_{\{m n\} \neq\{j\}} h_{m n} S_{m n j l}+h_{j l}\left(P_{j l}-P_{0}\right) \\
& i \hbar \dot{S}_{m n j l}= S_{m n j l}\left(\bar{E}_{m n}-\bar{E}_{j l}\right)+S_{m n j l} \\
& \times\left(\Sigma_{k \neq m, n} T_{k(m n)}-\Sigma_{k \neq j, l} T_{k(j l)}\right) \\
&+h_{m n} S_{0 j l}-h_{j l} S_{0 m n}^{*}
\end{aligned}
$$

where $j, k, l, m, n$ label the single particle levels in the active pairing space. The sign $s= \pm 1$ ensures that the matrix element of the expression (1) is positive. $N_{i_{k}}=N_{2}$ or $N_{i_{k}}=-N_{1}$ if the state $k$ will be located in the fragment 1 or in the fragment 2 after the scission, respectively. $T_{\gamma}$ are state dependent energy terms. The following notations are used:

$$
\begin{array}{cc}
\Delta_{k(0)}=\sum_{k^{\prime}} \kappa_{k^{\prime}(0)} G_{k k^{\prime}} ; & \Delta_{k(j l)}=\sum_{k^{\prime} \neq j, l} \kappa_{k^{\prime}(j l)} G_{k k^{\prime}} ; \\
\kappa_{k(0)}=u_{k(0)} v_{k(0)} ; & \rho_{k(0)}=\left|v_{k(0)}\right|^{2} ; \\
\kappa_{k(j l)}=u_{k(j l)} v_{k(j l)} ; & \rho_{k(j l)}=\left|v_{k(j l)}\right|^{2} ; \\
P_{0}=\left|c_{0}\right|^{2} ; & P_{j l}=\left|c_{j l}\right|^{2} ; \\
S_{0 j l}=c_{0} c_{j l}^{*} ; & S_{m n j l}=c_{m n} c_{j l}^{*} .
\end{array}
$$

The symbol $\Delta_{\gamma}$ gives the gap parameter. (The label $\gamma$ denotes here generically a specific configuration.) The variables that depend on the time through the generalized coordinates are the single particle densities $\rho_{\gamma}$, the pairing moment components $\kappa_{\gamma}$, the probabilities to have a given seniority configuration $P_{\gamma}$, and the moment components between configurations $S_{\gamma \gamma^{\prime}}$. The relations (4)-(7) are the well known time dependent paring equations for one seniority configuration previously deduced in Refs. [20,21]. These formulas are identical to the time dependent Hartree-Fock-Bogoliubov equations [22-24]. The symbol $h_{\gamma}$ denotes the Landau-Zener interaction, while $\bar{E}_{\gamma}$ and $T_{\gamma}$ are energy terms. The condition that $\sum_{\gamma} P_{\gamma}=1$ is implicitly ensured through Eqs. (8) and (9) because $\dot{P}_{0}+$ $\sum_{\gamma} \dot{P}_{\gamma}=0$.

It should be mentioned that the time dependent pairing equations supply an average of the magnitude of the dissipated energy obtained along the least action path as defined in Ref. [20],

$$
E_{0}^{*}=E_{0}-E_{0}^{0} ; \quad E_{j l}^{*}=E_{j l}-E_{j l}^{0},
$$


in the seniority zero and the seniority two configurations, respectively. We subtracted from the total potential energy of the nuclear system, its adiabatic value. It was already shown in Ref. [25] that the mean value of the dissipated energy becomes larger when the velocities of the generalized coordinates increase.

\section{Results}

In order to solve the equations of motion, we need energies of the single particle level scheme in the active pairing space and the velocities of passage through the avoided crossing regions. If the fission trajectory of the nuclear system is determined from the ground state of the compound system up to the scission as function of the internuclear distance between the two nascent fragments, the single particle energies can be calculated within a microscopic model while the velocity is given by the internuclear velocity. The least action principle provides a way to determine most likely the fission trajectory. The method is limited by the fact that no diffusive components of the nuclear shape evolution are taken into account. Two ingredients were required: the deformation energy and the inertia. The total energy of the nuclear system is computed in the framework of the macroscopic-microscopic method [26-28]. Within this method, the whole system is characterized by some collective coordinates that determine approximately the behavior of many other intrinsic variables. The generalized coordinates vary in time leading to a split of the system. The procedure is analogous to that used in more sophisticated models as the Hartree-Fock, where external constraints simulate the deformation $[29,30]$. In our calculations, the many-body wave function and the single particle energies are provided by the WoodsSaxon two-center shell model [15]. This model was widely used by the Bucharest group in the calculations addressing the cluster [31,32] and alpha decay [33-35], the fission [36-38], or the heavy element synthesis [3942]. The two center shell model is particularly appropriate for the characterization of scission regions [43]. For the inertia: the non-adiabatic cranking model [44], the Gaussian overlap approximation $[45,46]$, and the cranking approach [13] were used. We are interested to find a fission trajectory for the fragmentation ${ }^{90} \mathrm{Kr}+{ }^{144} \mathrm{Ba}$ (even-even), ${ }^{90} \mathrm{Rb}+{ }^{144} \mathrm{Cs}$ (odd-odd). Experimental data are available for this mass fragmentation [8] of the reaction ${ }^{233} \mathrm{U}\left(\mathrm{n}_{t h}, \mathrm{f}\right)$. By minimization in the framework of the least action principle, we obtained the most probable fragmentation, that corresponds to a heavy fragment with mass around 138. In our work, the fission path for the $144 / 90$ fragmentation is required. To adapt our least action trajectory, some simple assumptions are made. We rely on the results of Refs. [19,36] where the mass distribution of the fragments was relatively well reproduced by considering that the variation of the mass asymmetry is linear from the saddle configuration of the outer barrier up to the exit point. So, it is considered that under the rapid descent from the top of the second barrier different mass partitions are obtained. To obtain these different mass partitions, the simplest way is to vary the mass asymmetry parameter and the deformations of fragments. Once the fission path determined, we calculated the single particle energies in the active level pairing space. Our pairing

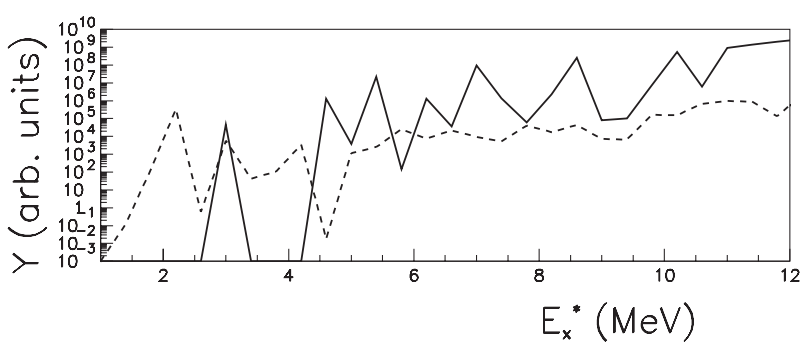

Figure 2. Full line: dependence of even even yield in arbitrary units $Y_{0}$ as function of the final excitation $E_{x}^{*}$ of the fission fragments. Dashed line: dependence of the odd-odd yields $Y_{2}$ as function of the excitation energy [17].

active space is constructed with 58 single particle levels around the Fermi energy. The avoided crossing regions that pertain to adjacent single particle levels characterized by the same good quantum numbers are identified in a manner similar to that of Ref. [47]. Several values of the internuclear velocity $v=\dot{R}$ are tested in order to solve the equations of motion: $5 \times 10^{2}, 8 \times 10^{2}, 10^{3}, 3 \times 10^{3}, 10^{4}$, $3 \times 10^{4}, 10^{5}, 3 \times 10^{5}, 10^{6}$, and $3 \times 10^{6} \mathrm{fm} / \mathrm{fs}$. The maximal velocity of $3 \times 10^{6} \mathrm{fm} / \mathrm{fs}$ is also close to the limit of validity of the model. This extreme velocity is compatible to a scission time of the order of $10^{-21} \mathrm{~s}$, used in Ref. [48] to describe the neutron emission within a time-dependent microscopic approach.

The equations of motion are solved and the probabilities to obtain different seniority configurations at scission together with their excitation energies are obtained as function of the internuclear velocity. In order to compare the theory with the experimental data, the previous dependencies were folded in order to eliminate the internuclear velocity. The probabilities to obtain eveneven yields or odd-odd ones in the final fragments as function of the excitation energy were calculated. The results obtained for the folded distributions $Y_{0}\left(E_{x}^{*}\right)$ (eveneven yields) and $Y_{2}\left(E_{x}^{*}\right)$ (odd-odd yields) are displayed in Fig. 2. The observed experimental trends exhibited in Fig. 4 of Ref. [8] for cold fission yields were reproduced. That is, at low excitation energy the odd-odd yields surpass the even-even ones and the even-even yields become larger for excitation energies larger that $3-4 \mathrm{MeV}$.

\section{Conclusions}

In this work, a microscopic model is proposed for the explanation of the odd-even effect in cold fission. This explanation is based on a mixing configuration mechanism that is produced during the fission process. This configuration mixing mechanism is obtained dynamically by solving a the generalized system of time dependent pairing equations, that include a pair-breaking effect. A first rule can be extracted from this model. The eveneven fission products cannot be obtained at zero excitation energies because of the existence of dynamical excitations produced in the avoided level crossing regions when the nuclear system deforms slowly.

Work supported by CNCS-UEFISCDI, project number PN-II-IDPCE-2011-3-0068. 


\section{References}

[1] W.N. Reisdorf, J.P. Unik, and L.E. Glendenin, Nucl. Phys. A 205, 348 (1973)

[2] H. Wohlfarth, W. Lang, H.-G. Clerc, H. Schrader, K.-H. Schmidt, and H. Dann, Phys. Lett. B 63, 275 (1976)

[3] C. Signarbieux, M. Montoya, M. Ribrag, C. Mazur, C. Guet, P. Perrin, and M. Maurel, J. Phys. Lett. 42, 437 (1981)

[4] M. Caamano, F. Rejmund, and K.-H. Schmidt, J. Phys. G 38, 035101 (2011)

[5] B. Jurado and K.-H. Schmidt, EPJ Web of Conferences 62, 07003 (2013)

[6] A. Tudora, F.-J. Hambsch, G. Giubega and I. Visan, Rom. Rep. Phys. 68, 571 (2016)

[7] H.-G. Clerc, in Heavy Elements and Related New Phenomena, edited by W. Greiner and R.K. Gupta (World Scientific, Singapore, 1999), p. 471

[8] E. Schwab, H.-G. Clerc, M. Mutterer, J. P. Theobald, and H. Faust, Nucl. Phys. A 577, 674 (1994)

[9] F.-J. Hambsch, H.-H. Knitter, and C. BudtzJorgensen, Nucl. Phys. A 554, 209 (1993)

[10] H.-H. Knitter, F.-J. Hambsch, and C. BudtzJorgensen, Nucl. Phys. A 536, 221 (1992)

[11] V. Avrigeanu, A. Florescu, A. Sandulescu, and W. Greiner, Phys. Rev. C 52, R1755 (1995)

[12] B. Jurado and K.-H. Schmidt, J. Phys. G. 42, 055101 (2015)

[13] D.L. Hill and J.A. Wheeler, Phys. Rev. 89, 1102 (1953)

[14] F. Gonnenwein, Phys. Procedia 47, 107 (2013)

[15] M. Mirea, Phys. Rev. C 78, 044618 (2008)

[16] M. Mirea, Phys. Lett. B 680, 316 (2009)

[17] M. Mirea, Phys. Rev. C 89, 034623 (2014)

[18] M. Mirea, Phys. Rev. C 83, 054608 (2011)

[19] M. Mirea, Phys. Lett. B 717, 252 (2012)

[20] S.E. Koonin, J.R. Nix, Phys. Rev. C 13, 209 (1976)

[21] J. Blocki, H. Flocard, Nucl. Phys. A 273, 45 (1976)

[22] B. Avez, C. Simenel and Ph. Chomaz, Phys. Rev. C 78, 044318 (2008)

[23] S. Ebata, T. Nakatsukasa, T. Inakura, K. Yoshida, Y. Hashimoto and K. Yabana, Phys. Rev. C 82, 034306 (2010)

[24] J.W. Negele, Rev. Mod. Phys. 54, 913 (1982)
[25] M. Mirea, L. Tassan-Got, C. Stephan, C.O. Bacri, P. Stoica, R.C. Bobulescu, Nucl. Phys. A 735, 21 (2004)

[26] J.R. Nix, Ann. Rev. Nucl. Sci. 22, 65 (1972)

[27] W.J. Swiatecki, S. Bjornholm, Phys. Rep. 4, 325 (1972)

[28] M. Brack, J. Damgaard, A.S. Jensen, H.C. Pauli, V.M. Strutinsky, C.Y. Wong, Rev. Mod. Phys. 44, 320 (1972)

[29] C. Simenel, A.S. Umar, Phys. Rev. C 89, 031601(R) (2014)

[30] G. Scamps, C. Simenel, D. Lacroix, Phys. Rev. C 92, 011602(R) (2015)

[31] M. Mirea, A. Sandulescu, D.S. Delion, Nucl. Phys. A 870, 23 (2011)

[32] M. Mirea, A. Sandulescu, D.S. Delion, Eur. Phys. J. A 48, 85 (2012)

[33] A. Sandulescu, M. Mirea, D.S. Delion, EPL 101, 62001 (2013)

[34] M. Mirea, Eur. Phys. J. A 51, 36 (2015)

[35] M. Mirea, Rom. J. Phys. 60, 156 (2015)

[36] M. Mirea, D.S. Delion, A. Sandulescu, Phys. Rev. C 81, 044317 (2010)

[37] M. Mirea, L. Tassan-Got, Centr. Eur. J. Phys. 9, 116 (2011)

[38] R. Budaca, A. Sandulescu, M. Mirea, Mod. Phys. Lett. A 30, 1550129 (2015)

[39] M. Mirea, D.S. Delion, A. Sandulescu, EPL 85, 121001 (2009)

[40] A. Sandulescu, M. Mirea, Eur. Phys. J. A 50, 110 (2014)

[41] D. Aranghel and A. Sandulescu, Rom. Rep. Phys. 68, 160 (2016)

[42] D. Aranghel and A. Sandulescu, Rom. J. Phys. 60, 147 (2015)

[43] F.A. Ivanyuk, S. Chiba, A. Arimoto, Phys. Rev. C 90, 054607 (2014)

[44] M. Mirea, J. Phys. G 43, 105103 (2016)

[45] A. Gozdz, K. Pomorski, M. Brack, E. Werner, Nucl. Phys. A 442, 26 (1985)

[46] A. Gozdz, K. Pomorski, M. Brack, E. Werner, Nucl. Phys. A 442, 50 (1985)

[47] M. Mirea, L. Tassan-Got, C. Stephan, C.O. Bacri and R.C. Bobulescu, Phys. Rev. C 76, 064608 (2007)

[48] M. Rizea, N. Carjan, Proc. Rom. Acad. Series A 16, $176(2015)$ 